\title{
Nonvascular pathways of aqueous humor outflow in the choroid of the human eye
}

\author{
Sabina Nogovitsina \\ Laboratory ultrastructural research \\ Research Institute of Clinical and \\ Experimental Lymphology - Branch of \\ the Institute of Cytology and Genetics, \\ Siberian Branch of the Russian \\ Academy of Sciences \\ Novosibirsk, Russian Federation \\ nogovitsina.niikel@gmail.com \\ Valeriy Chernykh \\ The academician S.N. Fyodorov \\ Federal State Institution National \\ Medical Research Center «Intersectoral \\ Research and Technology Complex \\ «Eye microsurgery» Ministry of Health \\ of the Russian Federation», \\ Novosibirsk Branch \\ Novosibirsk, Russian Federation \\ rimma@mntk.nsk.ru
}

\author{
Nataliya Bgatova \\ Laboratory ultrastructural research \\ Research Institute of Clinical and \\ Experimental Lymphology - Branch of \\ the Institute of Cytology and Genetics, \\ Siberian Branch of the Russian \\ Academy of Sciences \\ Novosibirsk, Russian Federation \\ n_bgatova@ngs.ru \\ Aleksandr Trunov \\ Science department \\ The academician S.N. Fyodorov \\ Federal State Institution National \\ Medical Research Center «Intersectoral \\ Research and Technology Complex \\ «Eye microsurgery» Ministry of Health \\ of the Russian Federation», \\ Novosibirsk Branch \\ Novosibirsk, Russian Federation \\ trunov1963@yandex.ru
}

\author{
Alena Eremina \\ Science department \\ The academician S.N. Fyodorov \\ Federal State Institution National \\ Medical Research Center «Intersectoral \\ Research and Technology Complex \\ «Eye microsurgery» Ministry of Health \\ of the Russian Federation», \\ Novosibirsk Branch \\ Novosibirsk, Russian Federation \\ sci@mntk.nsk.ru \\ Vladimir Konenkov \\ Laboratory of clinical immunogenetics \\ Research Institute of Clinical and \\ Experimental Lymphology - Branch of \\ the Institute of Cytology and Genetics, \\ Siberian Branch of the Russian \\ Academy of Sciences \\ Novosibirsk, Russian Federation \\ konenkov@soramn.ru
}

\begin{abstract}
The structural organization of lymphatic-like channels in the human eye choroid was studied by using immunohistochemistry and transmission electron microscopy. It was shown that these channels lining fibroblast-like cells that express markers of lymphatic endothelium. (Abstract)
\end{abstract}

Keywords - fibroblast-like cells, conjunctiva, choroid, lymphatic endothelium, lymphatic channels, human eye

\section{INTRODUCTION}

The question about presence of lymphatic outflow from human eye is uncertain until now. For a long time it was supposed that there were no lymphatics in the eye. But after the revelation of molecular markers of lymphatic endothelial cells it became possible to detect the lymphatic capillaries and vessels in the various structures of the human eye in normal and pathological conditions. Nowadays there are some data about lymphatic structures in the anterior eye segment, including ciliary body $[1,2]$. This structures possibly takes a part in unconventional pathway of aqueous humor drainage. However, other investigators were not able to reproduce these results $[3,4]$.

Currently, the question of the presence of lymphatic vessels in the posterior eye segment remains debatable. There are several researches about lymphatic structures in the choroid of the human eyes $[5,6]$. Despite the evidence of the existence of lymphatic vessels in the choroid, the structural organization of these lymphatic vessels is not described.

Therefore, the purpose of this research was to study the structural organization of the lymphatic pathways of aqueous humor outflow in the choroid.

\section{METHODS AND ALGORITHMS}

The object of study were fragments of enucleated eyes ( $\mathrm{n}$ $=10$ ), harvested from patients with normal intraocular pressure. For morphological study of the samples of the conjunctiva and the choroid were treated according to standard procedures for light and transmission electron microscopy. Paraffin sections were immunostained with the lymphatic specific endothelial markers Podoplanin (Monosan, Netherlands), Prox-1 (Covance, Germany), and LYVE -1 (Abcam, England), the vascular specific endothelial marker CD31 (Abcam, England), a marker for fibroblast growth factor receptors FGFR-1 (Abcam, England) and fibroblast marker TE-7 (Novus Biologicals, USA). Stained paraffin sections were examined with a light microscope "Leica DME» (Germany). Ultrathin sections were examined in an electron microscope «JEM 1400» (Japan). Morphometric processing and statistical analysis of the results were performed.

\section{RESULTS}

The presence of lymphatic vessels in conjunctiva was verified and it have typical organization, so we used conjunctiva as a control tissue.

With simultaneous immunohistochemical staining of conjunctiva and choroid samples using antibodies to markers of lymphatic vessels (LYVE-1, Prox-1, Pooplanin), in the structure of the conjunctiva revealed positively stained typical lymphatics, and positively stained lymphatic-like structures in the choroid. The choroid was found to contain lymphatic channels in choroidal stroma and layer of choriocapillaris, and lymphatic lacunae in the suprachoroid lamina (the transition zone between choroid and sclera). These channels and lacunae weren't typical lymphatic vessels. The channels were limited by narrow elongated cells, positively stained for markers of lymphatic vessels Prox-1, LYVE-1, Podoplanin and were shown negative CD31 staining; therefore we consider that this channels and lacunae are lymphatic structures.

Using transmission electron microscopy we identified that lymphatic channels did not have a typical vascular structure. We shown that these channels circumscribed by narrow process cells that have ultrastructural differences from 
lymphatic endothelial cells: they do not have stropic filaments, contain a greater volume density of the granular endoplasmic reticulum membranes, and a lower volume density of mitochondria and caveolae.

The cells lining the lymphatic channels are similar to fibroblasts, but differ from them in the lower bulk density of the granular endoplasmic reticulum and the lower content of fibrous structures in the microenvironment.

We carried out an immunohistochemical study of the samples using fibroblast markers FGFR-1 and TE-7. During immunohistochemical staining of samples using the TE-7 marker, rare positively colored elongated narrow cells were detected in the choroid of the human eye. As a result, staining of the same cells was noted as when staining for lymphatic endothelial markers. However, not all cells lining the channels studied by us were stained for fibroblast markers TE -7 and FGFR - 1

Considering that these cells have ultrastructural differences from fibroblasts and lymphatic endothelial cells, they express lymphatic endothelial markers and do not always have fibroblast markers, i.e. these cells are fibroblast-like cells.

\section{CONCLUSION}

Thus, in the structure of the choroid of the human eye, there are non-vascular pathways of aqueous humor outflow, represented by untypical lymphatic channels confined by fibroblast-like cells that have markers of the endothelium of the lymphatic vessels.

\section{REFERENCES}

[1] Y. H. Yücel M. G. Johnston, T. Ly, M. Patel, B. Drake, E. Gümüş, S. A. Fraenkl, S. Moore, Tobbia, D. Armstrong, E. Horvath and N. Gupta, "Identification of lymphatics in the ciliary body of the human eye: a novel "uveolymphatic" outflow pathway," Exp Eye Res., Vol. 89, pp. 810-819, 2009.J.

[2] M. Kim, M. G. Johnston, N. Gupta, S. Moore and Y. H. Yücel, "A model to measure lymphatic drainage from the eye," Exp Eye Res., Vol. 93, pp. 586-591, 2011.

[3] K. Birke, E. Lütjen-Drecoll, D. Kerjaschki and M. Birke, "Expression of podoplanin and other lymphatic markers in the human anterior eye segment," Invest Ophthalmol Vis Sci., Vol. 51, pp. 344-354, 2010.

[4] A. Kaser-Eichberger, F. Schrödl, A. Trost, C. Strohmaier, B. Bogner, C. Runge, K. Motloch, D. Bruckner, M. Laimer, S. L. Schlereth, L. M. Heindl and H. A. Reitsamer, "Topography of lymphatic markers in human iris and ciliary body,". Invest Ophthalmol Vis Sci., Vol. 56, pp. 4943-4953, 2015.

[5] M. E. Koina, L. Baxter, S. J. Adamson, F. Arfuso, P. Hu, M. C. Madigan and T. Chan-Ling, "Evidence for lymphatics in the developing and adult human choroid,” Invest Ophthalmol Vis Sci., № 56, Vol. 2, pp. 1310-1327, 2015.

[6] V.V. Chernykh, Yu.I. Borodin, N.P. Bgatova, S.R. Nogovitsina, I.A. Toporkov, A.V. Eremina, A.N. Trunov and V.I. Konenkov, "Lymphatic structures in the ciliary body and choroid of the human eye," Sovremennye tekhnologii v oftal'mologii, Vol. 3, pp. 268, 2016. (In Russ.) 